\title{
Análisis de los componentes físicos y antropométricos de jóvenes futbolistas chilenos desde la categoría Sub-13 a Sub-19 \\ Analysis of the physical and anthropometric components of young Chilean footballers from category Sub-13 to Sub-19 \\ Joel Barrera*, Luis Valenzuela Contreras**, Fernando Maureira Cid***, Hugo Sarmento*

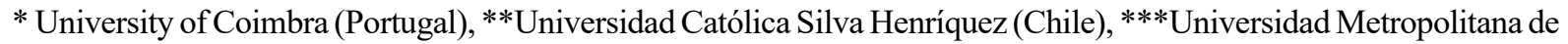 Ciencias de la Educación (Chile)
}

\begin{abstract}
Resumen. Objetivo: describir el perfil antropométrico y condicional de jóvenes futbolistas chilenos desde la categoría Sub-13 a Sub-19, proponiendo una batería de pruebas objetivas, que permita proporcionar una serie de indicadores validos de medición. Metodología: se aplicaron pruebas de campo de agilidad $20 \mathrm{~m}$ zigzag, aceleración $10 \mathrm{~m}$, velocidad $30 \mathrm{~m}$, Running based anaerobic sprint test (RAST), YoYo IR1 y salto contramovimiento (CMJ), sumado a las medidas antropométricas de altura y masa corporal. Resultados: Los resultados demostraron que los atletas de las categorías más jóvenes (Sub-13, Sub-14 y Sub-15) son más altos que los informados en otras publicaciones científicas. Además, el 73,2\% de los atletas nació en los dos primeros trimestres del año de selección, dejando en evidencia el efecto de edad relativa. Las categorías Sub-13 y Sub-14 presentaron el mayor número de diferencias al comparar sus medias en las diferentes variantes. Conclusión: La mayor presencia de pruebas objetivas permiten determinar el rendimiento físico de los jóvenes futbolista, esto a su vez consiente en crear un estándar de selección más objetivo y con un menor margen de error para los entrenadores. Palabras claves: Rendimiento, fútbol, selección, perfil.
\end{abstract}

\begin{abstract}
Objective: to describe the anthropometric and fitness profile of young Chilean soccer players from the Sub-13 to Sub-19 category, proposing a battery of objective tests, which allows providing a series of valid measurement indicators. Methodology: field tests of agility $20 \mathrm{~m}$ zigzag, acceleration $10 \mathrm{~m}$, speed $30 \mathrm{~m}$, running based anaerobic sprint test (RAST), Yo-Yo IR1 and countermovement jump (CMJ) were applied, added to anthropometric measurements of height and body mass. Results: The results showed that the athletes in the youngest categories (Sub-13, Sub-14 and Sub-15) are higher than those reported in other scientific publications. In addition, $73.2 \%$ of athletes were born in the first two quarters of the selection year, revealing the effect of relative age. Categories Sub13 and Sub-14 presented the greatest number of differences when comparing their means in the different variants. Conclusion: The greater presence of objective tests allows us to determine the physical performance of young soccer players, this in turn allows us to create a more objective selection standard and with a lower margin of error for coaches.
\end{abstract}

Keywords: Performance, football, selection, profile.

\section{Introducción}

Cuando se habla de identificación de talentos en el fútbol se refiere básicamente a la predicción del rendimiento que un atleta puede llegar a tener en este deporte, lo que resulta altamente complicado debido a la naturaleza dinámica del juego (Bergkamp, Niessen, den Hartigh, Frencken \& Meijer, 2019). Actualmente una gran cantidad de jóvenes sueña con ser futbolista de élite algún día, a pesar de ser un proceso altamente complejo donde menos del 10\% de los jóvenes atletas consigue ese objetivo (Grossmann \& Lames, 2015). En este contexto y a pesar que los programas de selección y desarrollo han ganado popularidad, no existe consenso en cómo se debe definir o identificar el talento y no existe un marco teórico uniformemente aceptado para guiar la práctica actual (Vaeyens, Lenoir, Williams, \& Philippaerts, 2008), estos mismos autores señalan que los programas de identificación y desarrollo de talentos deben ser dinámicos e interconectados, teniendo en cuenta el estado de madurez y el potencial para desarrollar, en lugar de excluir, a los niños a una edad temprana. De este modo la identificación y el desarrollo del talento exige un enfoque multidisciplinario y aquellos que trabajan directamente con los jóvenes requieren una sólida comprensión interdisciplinaria de las contribuciones disciplinarias claves (Cooke, Cobley, Till \& Wattie, 2010).

Fecha recepción: 02-01-20. Fecha de aceptación: 21-08-20 Fernando Maureira maureirafernando@yahoo.es
Hoy en día, el fútbol mundial se caracteriza por un mayor movimiento de jugadores entre países con cifras millonarias, lo que puede garantizar el éxito y/o la supervivencia deportiva y financiera de los clubes (Sarmento, Anguera, Pereira, \& Araujo, 2018). En Chile, las investigaciones de los últimos años se han centrado en evaluar diversos factores de los jóvenes futbolistas de manera aislada, como la valoración del pico de crecimiento (Roca, Vásquez, \& Valderas, 2017), los análisis de la composición corporal (Duarte, 2015; Hernández-Mosqueira, Fernandes, Fernandes, Retamales, Ibarra, Hernández-Vasquez, et al., 2013; Jorquera, Rodríguez, Torrealba, \& Barraza, 2012), además del análisis fisiológico del esfuerzo físico por puestos (Toro, 2001) y la valoración de la potencia y el índice de la fatiga (Roca, Vásquez, Valderas, Sepúlveda \& González, 2018). Estos elementos aislados de medición no entregan la información necesaria para determinar si existe una correcta selección de jóvenes futbolistas y con los instrumentos de evaluación más adecuados.

Por lo señalado anteriormente, es necesario generar estudios de análisis más nutridos de los diferentes elementos que interactúan para influir en la identificación y el desarrollo de talento en el fútbol, tal como indican Sarmento, et al., (2018). Considerar mayor cantidad de elementos que permita una selección más eficiente, sin desperdiciar talento que pueda estar oculto, en un nivel de madurez menor, al igual como ocurre en el baloncesto donde un estudio revelo la ausencia de jóvenes basquetbolistas que maduran tardíamente en los equipos de un rango de 14 y 15 años (Silva, Figueiredo, Moreira \& Malina, 2008), condición biológica 
que puede estar influenciado por aspectos demográficos, nutricionales y sociales (Bolívara, Sandovalc, Osorioa, Dibd, $\&$ Gallo, 2015) o a causa de un cuartil de nacimiento (efecto edad relativa) que pueda afectar en la selección de jóvenes deportistas (Couto, Macedo, Melo, Teoldo \& Fahel, 2009).

El efecto de edad relativa (RAE) es un fenómeno que presenta sus orígenes en el hockey canadiense por un estudio realizado por Barnsley, Thompson y Barnsley (1985). Varias investigaciones han demostrado que un número desproporcionado de jugadores juveniles de élite que rivalizan en una competición segmentada por edad nacen temprano en el año de inclusión, lo que aumenta sus posibilidades de selección, lo que se denomina efecto de edad relativa (Vincent \& Glamser, 2006). Por otra parte, Gutiérrez, Pastor, González, y Contreras (2010), propusieron identificar la existencia del efecto de edad relativa en niveles juvenil en los clubes de fútbol españoles de élite y aficionados, y también realizar un análisis que brinde información sobre cómo ha evolucionado este efecto en los últimos años en las categorías Sub-11, Sub-13, Sub-15 y Sub-18.

Tales condiciones biológicas y cronológicas, pueden afectar directamente en el rendimiento técnico-táctico en las categorías de formación, por un mayor rendimiento de las capacidades funcionales (Al Haddad, Simpson, Buchheit, Di Salvo \& Mendez-Villanueva, 2015; Unnithan, White, Georgiou, Iga \& Drust, 2012), sin embargo, las brechas que se producen en fuerza y poder se pierden cuando se comparan jugadores biológicamente maduros de diferente edad cronológica (Silva, et al., 2008).

Por otra parte, consideramos de igual manera diferenciar posicionalmente los resultados, ya que esto podría aportar mayor valor al manuscrito ya que, algunos autores destacan que existe una influencia de la posición de juego en las cualidades antropométricas y físicas de los jugadores adolescentes (Marqués et al., 2016), producto de su rol posicional el cual exige diferentes esfuerzo físico, el cual se puede utilizar para el desarrollo de programas específicos (Di Salvo et al., 2007) y con ello condicionar su perfil .

En cuanto a la literatura, los estudios a nivel europeo son abundantes, por lo cual es importante generar mayor número de investigaciones en el área de la selección de jóvenes futbolistas en américa, ya que es una fuente importante de exportación de talentos $\mathrm{y}$, además, es de donde han emergido alguno de los jugadores más talentosos de la historia del fútbol. Tradicionalmente en el fútbol, los jóvenes talentos fueron seleccionados de manera subjetiva en función del potencial para complementar el estilo de juego de su club (Sarmento, et al., 2018), esto genera la necesidad de optimizar el proceso actual de selección de los jóvenes futbolistas, con una estructura más sólida y más objetiva, que entregue mayor información que el simple análisis de un entrenador, el cual puede poseer un sesgó importante y con cifras de precisión observacional bajas (Nicholls \& Worsfold, 2016), además, los entrenadores de fútbol siempre buscan descubrir el próximo jugador estrella, sin invertir mucho recurso, tiempo y el esfuerzo necesarios en el desarrollo de un jugador (Dodd $\&$ Newans, 2018), muchas veces se presiona para la obtención del éxito inmediato (Hill \& Sotiriadou, 2016).

Por lo antes señalado, la presente investigación pretende describir el perfil de los jóvenes atletas chilenos de elite por medio de un conjunto de pruebas, lo que puede crear un parámetro de referencia en las diferentes categorías, con la finalidad de obtener una selección más objetiva y efectiva a nivel físico de los jóvenes talentos

\section{Métodos}

\section{Diseño de estudio}

Estudio descriptivo de corte transversal, donde se exponen las características de los componentes físicos y antropométricos de una muestra de jovenes futbolistas chilenos, con mediciones realizadas en un sólo momento (Maureira y Flores, 2018).

\section{Muestra}

Estuvo constituida por jóvenes futbolistas chilenos saludables de las categorías Sub-13, Sub-14, Sub-15, Sub-16, Sub-17 y Sub-19, los cuales fueron reclutados de dos equipos de la primera división del fútbol chileno $(\mathrm{N}=243$; edad $15.9 \pm 1.9$ años; masa corporal $=65.1 \pm 9.7 \mathrm{~kg}$; altura $=172.4 \pm 8.1$ $\mathrm{cm})$. Como criterio de exclusión fueron consideradas tres horas mínimas de entrenamiento y ser miembros inscritos en las competiciones oficiales. Por ser sujetos menores de edad se debió utilizar un consentimiento informado escrito de cada participante que debía ser firmado por el representante legal de los atletas. Los procedimientos experimentales de este estudio estaban adscritos con la Declaración de Helsinki.

\section{Instrumentos}

Para determinar la capacidad aeróbica y la recuperación intermitente se utilizará el Yo-Yo (IR1), el cual posee mayor aplicabilidad para niños $>13$ años (Schmitz, Pfeifer, Kreitz, Borowski, Faldum \& Brand, 2019) con una alta reproducibilidad y sensibilidad para determinar el rendimiento físico en el fútbol (Krustrup, Mohr, Amstrup, Rysgaard, Johansen, Steensberg, et al., 2003), dicho procedimiento consiste en recorrer una zona delimitada de 20 metros de ida y vuelta, la mayor cantidad de veces posible, a una velocidad progresivamente aumentada controlada por bleeps y de manera continua hasta llegar al punto de salida, en donde el deportista tendrá un período de recuperación de 10 segundos, en un trazo de 5 metros posterior a la línea de salida. El deportista debe procurar mantenerse en la prueba durante el mayor tiempo posible, en donde los criterios de exclusión consistirán la deserción voluntaria del deportista o cuando este de manera consecutiva no logre llegar al final de los 20 metros y se marcará la distancia recorrida en la última lanzadera exitosa para ser registrado y como el resultado alcanzado en la prueba. El audio será reproducido en un equipo de música portátil ((Máster-G® XWZ-451, China). Finalmente, con la distancia total recorrida que alcance cada deportista se procederá al tratamiento de la información y se determinara la capacidad aeróbica que posea el atleta mediante la siguiente formula (Bangsbo, Iaia \& Krustrup, 2008):

Yo-Yo teste IR1: VO2 $\max (\mathrm{ml} / \mathrm{min} / \mathrm{kg})=$ distancia IR1 (m) $\mathrm{x}$ $.0084+36.4$

La capacidad anaeróbica se determinará con la prueba RAST (Running based Anaerobic Sprint Test) la cual consiste en realizar una serie de seis sprint de 35 metros, con una pausa de 10 segundos entre cada sprint, esta prueba fue 
propuesto por Mackenzie (2005) y recomendado por este mismo autor para deportes de resistencia como el fútbol. Durante el proceso de ejecución de la prueba, se debe animar a los deportistas a realizar el máximo esfuerzo en cada uno de los sprint. Para dicho procedimiento se utilizaron fotocélulas (Smartspeed Pro, Additional Gate, fusion sport@), las cuales se posicionan al inicio y al final de los 35 metros, a una altura de un metro. El deportista se encontrará en posición bípeda, dos metros por detrás de la primera fotocélula y la salida será libre, los cinco sprint restantes se realizarán al término de la pausa de 10 segundos controlada por un profesor mediante un cronómetro (Casio HS-70W).

Por medio de los resultados obtenidos se determinó el índice de fatiga y la potencia media que se calculan mediante las siguientes formulas:

Índice de fatiga $=($ Potencia máx. - Potencia mín. $) /$ Tiempo total de los seis sprint

Potencia media: suma de los seis valores de potencia / 6

Para la evaluación de la aceleración y velocidad se utilizaran tres pares de fotocélulas (Smartspeed Pro, Additional Gate, fusion sport $($ ), al inició de la prueba, a los $10 \mathrm{~m}$ y a los $30 \mathrm{~m}$, parámetros que ya han sido utilizados en evaluaciones anteriores en futbolistas (Malý, Zahálka, Hráský, Mala, Izovská, Bujnovský, et al., 2015). Los jugadores comenzarán la prueba en posición bípeda a una distancia de dos metros antes de la primera fotocélula. Para este procedimiento los jugadores deberán realizar tres sprint, considerando una recuperación pasiva de tres minutos entre cada uno de ellos. Cada procedimiento será estimado para ambas pruebas $10 \mathrm{~m}$ y $30 \mathrm{~m}$ respectivamente con la finalidad de optimizar los tiempos de ejecución, de las cuales se considerará el mejor tiempo de ejecución registrado por cada atleta.

La evaluación de la agilidad se llevara a cabo por medio de la prueba en Zig-Zag propuesto por Little y Williams (2005), el cual consiste en realizar un recorrido de cuatro secciones de cinco metros con un ángulo de $100^{\circ}$ y un recorrido total de $20 \mathrm{~m}$. Está prueba se recomienda, debido a que utiliza constantes aceleraciones, frenos y cambios de dirección tal como ocurre en la práctica deportiva. Los atletas deberán estar en posición bípeda un metro antes de la posición del inicio de la prueba donde se instalará el primer par de fotocélulas y el segundo al final del recorrido, luego se dará una señal sonora para que los jugadores comiencen su recorrido, el cual podrán realizar en tres oportunidades con una recuperación pasiva de tres minutos entre cada prueba. La prueba se deberá realizar al menos 48 horas después del último juego o de un entrenamiento intenso. Los atletas tendrán la posibilidad experimentar previamente la prueba con pausas de dos minutos entre cada pasada para evitar un desgaste previo a la evaluación formal. Para esta evaluación se utilizó fotocélulas (Smartspeed Pro, Additional Gate, fusion sport $($ ).

La fuerza del tren inferior se evaluara mediante el salto vertical contramovimiento $(\mathrm{CMJ})$, que permite analizar la potencia de piernas de los atletas en el proceso completo de estiramiento acortamiento (Hernández \& Garcia, 2014). Este procedimiento consiste en determinar el tiempo de vuelo y altura del atleta desde que se despega de la plataforma de salto y vuelve a entrar en contacto con la misma. El procedi- miento consiste en: posicionar al atleta sobre la plataforma en posición bípeda y los pies separados a la anchura de los hombros, a la señal el deportista deberá realizar una flexión de rodillas aproximada de $90^{\circ} \mathrm{y}$ de manera inmediata un salto máximo en la vertical que se encuentra, durante la etapa de vuelo el deportista no debe flexionar las caderas o las rodillas, ya que para que el salto sea válido se deben mantener extendidas durante el vuelo. El salto se debe ejecutar con las manos del deportista en la cintura y no podrá retirarlas de esa posición durante la ejecución del procedimiento, con la finalidad de obtener un resultado que refleje solo la fuerza de los miembros inferiores sin el aporte que pueda generar el impulso de los brazos. Cada atleta tendrá la posibilidad de realizar tres saltos con un periodo de recuperación de al menos 30 segundos entre cada uno de ellos, con el objeto de no mermar el rendimiento entre cada uno de los saltos. Antes del inicio de la prueba se explicará el procedimiento y la correcta ejecución del procedimiento con la finalidad de evitar errores en su ejecución y optimizar de esa manera el tiempo al instante de recoger los datos. Para dicho procedimiento se utilizará una plataforma de salto marca Smartspeed Pro, Additional Gate, fusion sport@). La potencia se calculó mediante la fórmula propuesta por Sayers, Harackiewicz, Harman, Frykman, y Rosenstein (1999):

Potencia $=51.9 \times$ CMJ altura $(\mathrm{cm})+48.9 \times$ peso corporal (kg) -2007

Finalmente, para la medición de la altura y el peso se utilizó un estadiómetro fijado a la pared $\left(360^{\circ}\right.$ Wireless Seca 284 dp, Alemania) y para la obtención del peso total se utilizó una balanza digital balanza (Avanutri modelo BL MB con una división de 50 gr).

\section{Procedimiento}

Los resultados de las variables físicas se obtuvieron durante la temporada deportiva 2018-2019. Los futbolistas previamente a cada prueba debían realizar un calentamiento que era dirigido por el preparador físico de la categoría, el cual se ajustaba a las demandas de las pruebas a realizar. En las pruebas de aceleración, velocidad, agilidad y salto contramovimiento (CMJ), los atletas realizaron tres intentos de los cuales se consideró el mejor de ellos. En el caso de Running based anaerobic sprint test (RAST) y Yo-Yo (IR1), se consideraba la ejecución completa del proceso. Cabe señalar que las mediciones en un grupo de la categoría Sub-17 realizaron la prueba de agilidad en yerba natural, a diferencia del resto de categorías, lo cual puede influenciar en el patrón de carrera (López-Gómez et al., 2020).

El efecto de edad relativa (RAE) se analizó por medio de la recolección de información proporcionada por los clubes y su posterior clasificación según mes de nacimiento.

\section{Análisis estadístico}

Los primeros indicadores descriptivos que se extraerán de los datos obtenidos serán las medias, desvíos estándar y frecuencias. Posteriormente se realizará un análisis comparativo entre dos categorías Sub-13-Sub-14, Sub-15-Sub-16 y Sub-17-Sub-19 para determinar si existían diferencias significativas entre ellas. La normalidad de la distribución de datos se verificó mediante la prueba de Kolmogorov-Smirnov, lo que derivó en la utilización de la prueba no paramétrico $U$ de 
Mann-Whitney, para alcanzar los objetivos propuestos en el estudio (Maureira, 2017). Los datos serán almacenados en una planilla Excel y todos los análisis estadísticos se realizaron utilizando las estadísticas de IBM SPSS para Windows (V23.0, Armonk, NY: IBM. Corp.) y el nivel de significancia se estableció en $\mathrm{p}<.05$.

\section{Resultados}

Las características antropométricas de los participantes en relación con el peso y la altura se presentan en la tabla 1 y los resultados de las comparaciones entre pares de categorías Sub-13 y Sub-14, Sub-15 y Sub-16, Sub-17 y Sub-19, de todas las variables analizadas se presentan en la tabla 8 .

Al comparar los diferentes grupos en relación con la altura y la masa corporal entre las categorías Sub-13 y Sub-14 solo se apreciaron diferencias en la variable peso. Por su parte, las categorías Sub-15 y Sub-16 presentaron diferencias en la altura de los atletas. Finalmente, en las categorías Sub-17 y Sub-19 no se observaron diferencias entre estas variables.

Por otra parte, al analizar las medias

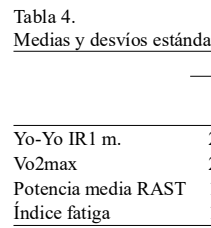
\begin{tabular}{ll} 
Potencia media RAST & 10 \\
Indice fatiga & 10 \\
\hline
\end{tabular} por posiciones utilizadas en el campo, los atletas más altos son los porteros, seguido por los defensores centrales, defensores laterales, delanteros y finalmente los mediocampistas (tabla 2).

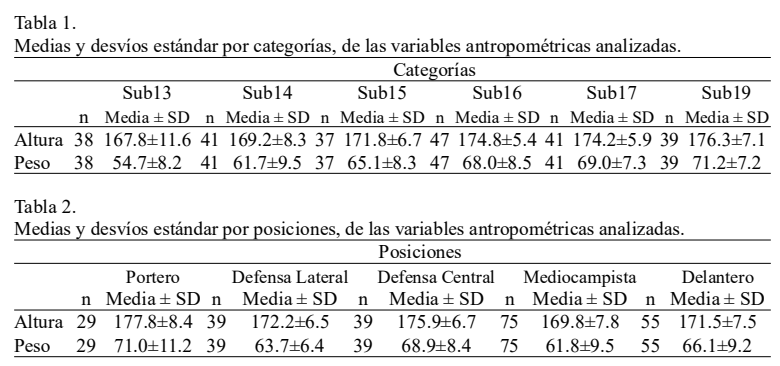

En relación con el efecto de edad relativa en el total de categorías estudiadas se observó que, de los 243 atletas, la distribución fue de $51.0 \%$ clasificados en el cuartil 1 (eneromarzo), 22.2\% en cuartil 2 (abril-junio), 15.2\% cuartil 3 (julioagosto) y $11.5 \%$ cuartil 4 (septiembre-diciembre). Lo que crea una distribución por categorías que se puede apreciar en la tabla 3. Dicha distribución deja de manifiesto un dominio por parte de los atletas nacidos en el cuartil 1 con el $51.0 \%$ de los atletas.

\begin{tabular}{|c|c|c|c|c|c|}
\hline Categorías & Q1 & Q2 & Q3 & Q4 & Total \\
\hline Sub13 & $20(52.6 \%)$ & $6(15.8 \%)$ & $9(23.7 \%)$ & $3(7.9 \%)$ & $38(100 \%)$ \\
\hline Sub14 & $23(56.1 \%)$ & $7(17.1 \%)$ & $6(14.6 \%)$ & $5(12.2 \%)$ & $41(100 \%)$ \\
\hline Sub15 & $16(43.2 \%)$ & $12(32.4 \%)$ & $3(8.1 \%)$ & $6(16.2 \%)$ & $37(100 \%)$ \\
\hline Sub16 & $25(53.2 \%)$ & $10(21.3 \%)$ & $6(12.8 \%)$ & $6(12.8 \%)$ & $47(100 \%)$ \\
\hline Sub17 & $18(43.9 \%)$ & $9(22.0 \%)$ & $7(17.1 \%)$ & $7(17.1 \%)$ & $41(100 \%)$ \\
\hline Sub19 & $22(56.4 \%)$ & $10(25.6 \%)$ & $6(15.4 \%)$ & $1(2.6 \%)$ & $39(100 \%)$ \\
\hline TOTAL & $124(51.0 \%)$ & $54(22.2 \%)$ & $37(15.2 \%)$ & $28(11.5 \%)$ & $243(100 \%)$ \\
\hline
\end{tabular}

El análisis de las capacidades físicas inicia con la estimación del VO2max., donde los resultados obtenidos en un contexto general fueron de $50.6 \pm 5.1 \mathrm{ml} / \mathrm{min} / \mathrm{kg}$. (tabla 4). En tanto, las categorías Sub-13 y Sub-14 presentaron una diferencia significativa entre ellas. En relación con, el nivel de VO2max. por posiciones reflejo que los porteros obtuvieron la media más baja, seguido de los delanteros, mediocampistas, defensores centrales y finalmente los defensores laterales (tabla 5).

Por otro lado, la prueba del RAST entregó resultados medios de potencia de $568.8 \pm 159.9$ watts, con valores ascendentes a medida que aumentan las categorías, además los resultados por posición los resultados más altos correspondieron a los defensores centrales y los más bajos fueron los mediocampistas. En relación con el índice de fatiga se observan diferencias significativas entre las categorías Sub-13 y Sub-14, al igual que en las categorías Sub-15 y Sub-16. Por último, al comparar las categorías Sub-17 y Sub-19 no se apreciaron diferencias entre estas categorías. En el análisis por posición, el mejor rendimiento en relación con el índice de fatiga lo obtuvieron los mediocampistas y el peor los delanteros, pera cabe señalar que tanto los delanteros como los defensores centrales tuvieron valores de potencia media mayor que los mediocampistas.

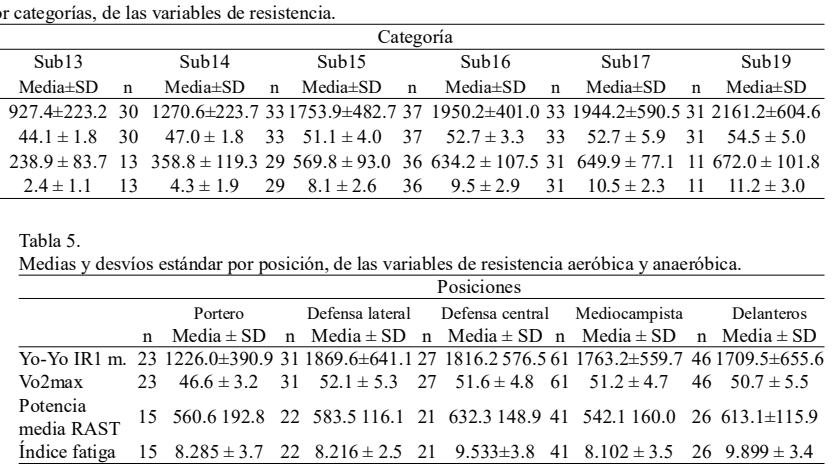

En la variable de aceleración en $10 \mathrm{~m}$ (Tabla 6) la comparación de las medias entre Sub-13 y Sub-14 no arrojo diferencias estadísticamente significativas, misma situación que ocurrió con la comparación de las categorías Sub-15, Sub-16 y Sub-17, Sub-19. Por otra parte, el rendimiento según posiciones evidenció que los mejores tiempos correspondieron a los defensores laterales y los peores a los porteros (Tabla 7).

Los resultados de la prueba de velocidad en $30 \mathrm{~m}$ mostraron diferencias estadísticamente significativas entre las categorías Sub-13 y Sub-14. Por su parte, en las categorías Sub-15 y Sub-16 no se presentaron diferencias significativas, misma situación que ocurrió entre las categorías Sub-17 y Sub-19. En tanto, en análisis por posiciones demostró que los más rápidos fueron los defensores laterales y los más lentos los porteros.

Tabla 6.

Medias y desvíos estándar por categorías, de las variables de aceleración, velocidad, agilidad y salto. \begin{tabular}{llllll}
\hline \multicolumn{7}{c}{ Categorías } \\
\hline Sub13 & Sub14 & Sub15 & Sub16 & Sub17 & Sub19
\end{tabular} Media \pm SD n Media \pm SD n Media \pm SD n Media \pm SD n Media \pm SD n Media \pm SD

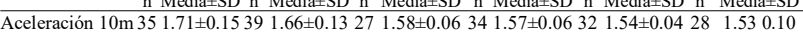
Velocidad 30m $354.45 \pm 0.38394 .26 \pm 0.30274 .13 \pm 0.17344 .04 \pm 0.19323 .98 \pm 0.1228 \quad 3.92 \pm 0.28$ Agilidad $20 \mathrm{~m} \quad 356.09 \pm 0.36395 .92 \pm 0.3027 \quad 5.66 \pm 0.21 \quad 385.70 \pm 0.1819 \quad 5.65 \pm 0.18 \quad 28 \quad 5.47 \pm 0.24$ Altura CMJ $\quad 3429.61 \pm 5.53832 .40 \pm 5.23433 .45 \pm 6.23836 .79 \pm 6.13738 .11 \pm 6.62938 .40 \pm 5.10$ Potencia CMJ $\quad 342155 \pm 578382675 \pm 656342882 \pm 622 \quad 383174 \pm 55737 \quad 3314 \pm 512 \quad 293447 \pm 507$

Tabla 7.

Medias y desvíos estándar por posición, de las variables de aceleración, velocidad, agilidad y salto. Posiciones

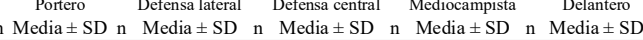

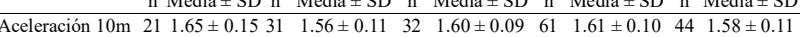

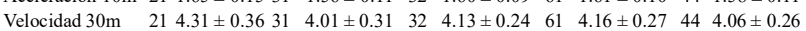

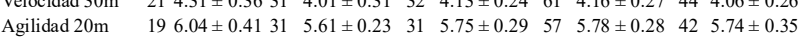

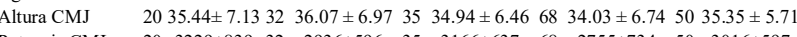

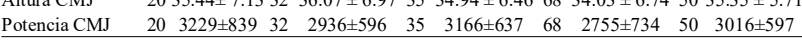


Los resultados obtenidos en agilidad muestran diferencias significativas entre las categorías Sub-13 y Sub-14, misma situación que ocurre con las categorías Sub-17 y Sub-19. Las categorías Sub-15 y Sub-16 no mostraron diferencias. En relación con las posiciones que utilizan los atletas los mejores resultados fueron obtenidos por los defensores laterales y los más bajos por los porteros.

\begin{tabular}{|c|c|c|c|}
\hline \multirow{3}{*}{ analizada. } & \multicolumn{3}{|c|}{ Categoría } \\
\hline & sub13-sub14 & sub15-sub16 & sub17-sub19 \\
\hline & Comparación de med & mparación de me & Comparación de medias \\
\hline Altura & - & 0.019 & - \\
\hline Peso & 0.001 & - & - \\
\hline Yo-Yo IR $1 \mathrm{~m}$. & 0,000 & - & - \\
\hline Vo2max & 0,000 & - & - \\
\hline Potencia media RAST & 0.012 & 0.014 & - \\
\hline Índice Fatiga & 0.008 & 0.049 & - \\
\hline Aceleración $10 \mathrm{~m}$ & - & - & - \\
\hline Velocidad 30m & 0.032 & - & - \\
\hline Agilidad $20 \mathrm{~m}$ & 0.030 & - & 0.016 \\
\hline Altura CMJ & 0.022 & - & - \\
\hline Potencia CMJ & - & - & - \\
\hline
\end{tabular}

Por último. la evaluación final realizada en este estudio fue en la altura CMJ, donde solo se aprecian diferencias significativas en las categorías Sub-13 y Sub-14. Con relación a las posiciones de los atletas, los resultados demostraron que los atletas con un mejor rendimiento en esta variable fueron los defensores laterales y los con el registro más bajo los mediocampistas.

\section{Discusiones}

El presente estudio ha sido el resultado de la intención de crear un perfil para los jugadores jóvenes de fútbol chileno. por medio de un conjunto de pruebas físicas. De esta manera, los resultados obtenidos. pueden resultar como medio de parámetro para la selección de nuevos atletas que ingresen al fútbol formativo en diferentes equipos en estos rangos etarios.

En relación con la altura. lo datos obtenidos en las categorías Sub-13, Sub-14 y Sub-15 son más altos que los obtenidos por Malina, Figueiredo y Coelho-E-Silva (2017), en un estudio que consistió en una revisión de los artículos publicados en esta materia sobre futbolistas, desde el año 1978 al año 2015. Los hallazgos que obtuvieron indicaron valores de altura entre $151.3 \mathrm{~cm} 11$ a 12 años y $165.0 \mathrm{~cm} 13$ a 14 años, que equivalen a los atletas de las categorías Sub-13, Sub-14 y Sub-15, donde los resultados de este estudio demuestran una altura mayor de $16.5 \mathrm{~cm}, 4.2 \mathrm{~cm}$ y $6.7 \mathrm{~cm}$ respectivamente. Sin embargo, en los siguientes escalones etarios los resultados tienden a ser similares. Con relación con la masa corporal los resultados entre los rangos de 11 a 12 años y de 13 a 14 años, son similares a los obtenidos en relación con la altura entre las categorías Sub-13, Sub-14 y Sub-15 con un peso mayor de los futbolistas de este estudio de $12.1 \mathrm{~kg}, 7.2 \mathrm{~kg}$ y $10.6 \mathrm{~kg}$ respectivamente, mientras que en las categorías mayores Sub-16, Sub-17 y Sub-19 los datos tienden a ser equiparados entre ambos estudios. Lo que deja de manifiesto un interés por reclutar a temprana edad atletas de mayor altura, lo que puede traer consigo dos elementos relevantes: primero, selección y retención que favorece a los atletas más altos producto de un nivel de maduración más avanzado o normales y segundo, el abandono por parte de los maduradores tardíos a temprana edad (Malina et al., 2017). Por otra parte. puede ser necesario crear mecanismos de protección por parte de los entrenadores o a nivel administrativo deportivo para aquellos atletas más pequeños y habilidosos durante los años de adolescencia (Figueiredo, Silva, Cumming \& Malina. 2010). Estas problemáticas quedan de manifiesto en el presente estudio, en las diferentes comparaciones, ya que las categorías que presentaron diferencias significativas en los resultados fueron la Sub-13 y Sub-14 las cuales se encuentran literalmente dividida por el pico de velocidad de crecimiento lo que puede favorecer a los jugadores de la categoría Sub-14 en comparación de los Sub-13 a casusa de un mayor desarrollo biológico (Malina et al., 2017).

Con relación a las posiciones y desde un análisis general que considera el total de la muestra, al ser contrastado los resultados con los obtenidos por Bernal-Orozco et al., (2020) con jugadores Sub-17 de México, podemos observar que se aprecia la misma tendencia en altura, donde se intenta reclutar porteros y defensores centrales de mayor envergadura, los que a su vez también son los más pesados, lo cual es una tendencia en fútbol moderno.

En directa relación con lo planteado anteriormente, el efecto de edad relativa se encuentra presente en la muestra de jóvenes futbolistas evaluados. Estos hallazgos evidenciaron un total 124 sujetos nacidos en la primera mitad del año correspondientes al $73.2 \%$, donde los nacidos en el primer trimestre correspondieron al $51.0 \%$ y en el segundo trimestre un $22.2 \%$, lo que demuestra semejanza con los hallazgos en atletas alemanes. españoles y australianos (Del Campo, Vicedo, Villora \& Jordan, 2010; Skorski, Skorski, Faude, Hammes \& Meyer, 2016; Van den Honert, 2012). Esto se debe a que la selección de atletas nacidos en la primera mitad del año se considera como un elemento importante para alcanzar el éxito juvenil (Augste \& Lames. 2011). Como consecuencia, las posibilidades de promoción deportiva son menores en los jóvenes nacidos en los últimos meses del año deportivo, debido a su menor grado de madurez física, mental y emocional (González, 2007). Esto podría llevar a que los jugadores nacidos en ciertos años tengan un camino restringido en el juego de élite, lo que lleva a la deserción entre este grupo, tal como indica Van den Honert (2012), y con ello la perdida de potenciales talentos para el futuro de cada club. Esto podría ser un error si se consideran los resultados obtenidos por Ostojic et al., (2014), los cuales demostraron que el $60.1 \%$ de los maduradores tardíos alcanzaron el fútbol de élite.

En tanto, los resultados relacionados con el consumo de oxígeno al compararlos con los obtenidos por Piqueras, Malavés y López (2010), son levemente inferiores, pero de igual modo que en el presente estudio, no reflejaron diferencias al comparar los grupos de 15 a 18 años. Por otra parte, un estudio realizado por Deprez et al., (2014), con atletas de élite y sub-élite con categorías Sub-13, Sub-15 y Sub-17, obtuvo en los futbolistas jóvenes valores más altos en la categoría Sub-13: 343 m; Sub-15: 65 m, mientras que en la Sub-17 los resultados de los futbolistas de este estudio fueron levemente mayores por $10 \mathrm{~m}$. Sin embargo, al contrastarlos con los datos obtenidos por Deprez, Fransen, Lenoir, Philippaerts y Vaeyens (2015), al estudiar las categorías de la Sub-15, Sub-17 y Sub-19, la brecha es aún mayor 271 m, 460 m y 314 $\mathrm{m}$, respectivamente. En este contexto, las diferencias que se 
pueden haber observado en relación con estos estudios, puede deberse a la cantidad de veces que los atletas fueron sometidos a la prueba durante un corto periodo de tiempo, lo que puede ayudar a la familiarización y adaptación del atleta hacia el esfuerzo y de este modo obtener mejores resultados. En el caso de este estudio, cabe señalar que los atletas solo fueron sometidos a esta prueba en una oportunidad. Con relación al rendimiento por las posiciones que los jugadores utilizan en el campo, los resultados del presente estudio concuerdan con los obtenidos por Rebelo et al., (2013) en que los jugadores de peor rendimiento son los porteros, seguido por lo delanteros, mientras que los mejores rendimientos se inclinaron por los mediocampistas y defensores laterales, los cuales recorren durante el juego formal la mayor distancia $12.0 \mathrm{~km}$ y $11.7 \mathrm{~km}$ respectivamente (Di Salvo et al., (2013), lo que en cierto modo podría explicar el rendimiento en esta prueba.

Por otra parte, los datos obtenidos en la prueba del RAST, al ser contrastados con los obtenidos por Cipryan y Gajda (2011), con jóvenes futbolistas Sub-19, evidenciaron un mejor rendimiento en la variable relacionada al índice de fatiga, que los atletas de este estudio (8.2 \pm 2.08$)$. Además, en relación con la producción de potencia media alcanzada por esta misma categoría en este estudio $(672.0 \pm 101.8 \mathrm{w})$ fue mayor en $72.4 \mathrm{w}$, a los datos obtenidos por este mismo autor (599.6 $\pm 66.25 \mathrm{w})$. En tanto, al comparar los datos obtenidos por Andrade et al., (2015), con atletas Sub-20 (682.4 \pm 80.2 w), los valores se encuentran levemente por encima de los obtenidos en el presente estudio en $10.4 \mathrm{w}$. Finalmente, al comparar los resultados de Hazir, Kose y Kin-Isler (2018), con futbolistas aficionados entre 14 y 17 años, obtuvo un valor medio (366.9 $\pm 84.2 \mathrm{w})$, menor a los obtenidos en las categorías Sub-15 (203w), Sub-16 (268w) y Sub-17 (283w) que corresponden a esos rangos etarios, al igual que los resultados obtenidos por Burgess, Holt, Munro y Swinton (2016) con atletas amateur ( $24 \pm 3$ años) los cuales alcanzaron una potencia media de $596 \pm 86 \mathrm{w}$, por debajo $76 \mathrm{w}$ a la obtenida por la categoría Sub-19 (672.0 101.8w). Estas diferencias en los resultados publicados con jugadores aficionados nos llevan a concluir que, el entrenamiento de alto nivel en jóvenes futbolistas puede generar adaptaciones que optimicen el rendimiento en este tipo de pruebas en comparación con jugadores amateurs, ya que tal como lo señala Rebelo et al., (2013), los jugadores de élite tienden a presentar más años de experiencia futbolística y horas de entrenamiento durante la temporada en relación con los jugadores que no son de élite.

A su vez, los datos obtenidos en la prueba de agilidad al ser comparados con los obtenidos por Kunrath, Gonçalves, Teoldo y Barbosa (2017), con atletas Sub-11, Sub-13 y Sub15 Fluminense Football Club (Rio de Janeiro, Brasil), evidenciaron que los resultados de este estudio en la categoría Sub-13 (6.09 $\pm .36 \mathrm{~s})$ son inferiores (.271 s) a los obtenidos por los autores antes señalados $(5.819 \pm .216 \mathrm{~s})$. Sin embargo, en la categoría Sub-15 (5.66 $\pm .21 \mathrm{~s})$ los resultados obtenidos en este estudio son levemente (.05 s) mejores $(5.710 \pm .179 \mathrm{~s})$. Esta situación podría deberse a la organización de los atletas brasileños, los cuales se clasifican cada dos años en una categoría según la clasificación del estudio analizado, a diferencia de lo que ocurre en Chile, donde cada categoría corresponde a un año calendario específico. Por otro lado, los datos obtenidos con jugadores profesionales entre 18 y 36 años de la primera y segunda división de la liga inglesa de las temporadas 2002-2003 declaro resultados en esta materia de $5.34 \pm .20 \mathrm{~s}$ (Little \& Williams, 2003), los cuales son levemente inferiores (.13 s) a los obtenidos por las categorías Sub-19 de

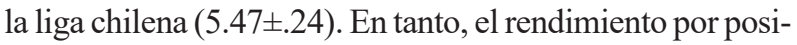
ciones con relación a un estudio con atletas Sub-19 de élite (Rebelo et al., 2013), las concordancias indican similitud en los rendimientos más bajos que corresponden a los porteros y mediocampistas, mientras que los más agiles tienden hacer los delanteros y defensores laterales, lo cual puede estar condicionado por su rol posicional (Di Salvo et al., 2007).

Con relación a la prueba de aceleración $10 \mathrm{~m}$ en línea recta, al ser comparada con los datos obtenidos por SilvaJunior et al., (2011), en un estudio realizado con 143 atletas del equipo Fluminense Football Club, entre las categorías Sub-15, Sub-17 y Sub-20 obtuvieron resultados de 1.84 \pm .12 s, $1.861 \pm .92 \mathrm{~s}$ y $1.74 \pm .06 \mathrm{~s}$ respectivamente, los cuales son inferiores a los alcanzados por los atletas de este estudio entre las categorías Sub-15 (.26 s), Sub-17 (.32 s) y Sub-19 $(.33 \mathrm{~s})$, estas diferencias son aún mayor si se comparan con los obtenidos por Little y Williams (2003), quien con atletas profesionales obtuvo una media de $1.83 \pm .08 \mathrm{~s}$. Finalmente al comparar los datos obtenidos por Kunrath, et al., (2017), en

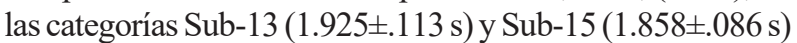
son inferiores a los obtenidos en el presente estudio en estas categorías Sub-13 (.21 s) y Sub-15 (.27 s) respectivamente. Estos resultados evidencian un mejor rendimiento en los jóvenes futbolistas chilenos, lo que se puede deber a las diferentes metodologías de trabajo aplicadas en la formación o a una diferencia en función a la tecnología a disposición para el control de esta prueba.

Por otro lado, en la prueba de velocidad de $30 \mathrm{~m}$. los datos obtenidos por Silva-Junior et al. (2011) con atletas brasileños Sub-15, Sub-17 y Sub-20, donde el mejor tiempo declarado fue de la categoría Sub-20 con 4.14 \pm .12 s. los datos de los futbolistas chilenos mejor registrados correspondieron a la categoría Sub-19 con un tiempo medio de 3.92 \pm .28 s. lo que crea una diferencia entre en el rendimiento de $.22 \mathrm{~s}$. Además, en las categorías Sub-13 y Sub-15 evaluadas por Kunrath et al., (2017), alcanzaron una media de $4.65 \pm .28 \mathrm{~s} \mathrm{y}$ $4.48 \pm .22 \mathrm{~s}$ respectivamente, por debajo de los valores de los futbolistas chilenos que alcanzaron en estas mismas categorías medias de $4.45 \pm .38 \mathrm{~s}(.20 \mathrm{~s})$ y $4.13 \pm .17 \mathrm{~s}(.35 \mathrm{~s})$ respectivamente. La prueba de $30 \mathrm{~m}$ coincide con los resultados de agilidad al posicionar a los porteros con el rendimiento más bajo, seguido por los mediocampistas y en el mejor rendimiento se encuentran los defensores laterales y delanteros, al igual que los resultados presentados por (Rebelo et al., 2013), mientras que los resultados presentados por Marques et al., (2016) posiciono a los delanteros con el mejor rendimiento en esta prueba. Lo cual puede deberse a los requerimientos específicos del juego, ya que los delanteros y defensores laterales son los que recorren mayor distancia a velocidades mayores a los $23 \mathrm{~km} / \mathrm{h}$ (Di Salvo et al., 2007).

Finalmente, los datos obtenidos en la prueba de salto CMJ, las categorías Sub-15 y Sub-17 del presente estudio, presentaron medias levemente inferiores en relación a la potencia y la altura del estudio realizado por Silva-Junior et al., (2011), 35.6 $\pm 4.3 \mathrm{~cm}(2802.8 \pm 469.7 \mathrm{w}) 39.0 \pm 5.0 \mathrm{~cm}$ 
$(3367.0 \pm 412.7 \mathrm{w})$ respectivamente, cifras que se acrecientan al comparar la Sub-19 con la Sub-20 de este mismo autor la cual alcanzo $42.6 \pm 3.9 \mathrm{~cm}(3802.0 \pm 363.5)$. Del mismo modo, al contrastar los resultados obtenidos en un estudio de intervención con relación a las mejoras que produce el entrenamiento pliométrico versus el entrenamiento de carga de potencia óptima en jugadores portugueses Sub-19 antes de la aplicación de los diferentes planes de entrenamientos, las diferencias entre estas categorías eran de $.16 \mathrm{~cm}$ a favor de los atletas portugueses, cifra que se acrecentó tras los diferentes programas de entrenamiento a $4.4 \mathrm{~cm}$. estos datos sugieren la aplicación de planes específicos de tren inferior para el mejoramiento en esta prueba y en la prueba de velocidad en línea recta (Ribeiro, et al.. 2019). En tanto, Los Arcos y Martins (2018) evaluaron el rendimiento por posiciones de los jugadores de elite de un equipo B de España, los cuales presentaron diferencias en relación con la altura de los saltos, dado que los delanteros profesionales son los que alcanzan un mejor rendimiento, seguido por los centrales y dejando en último lugar a los defensores laterales, que en el presente estudio obtienen el mejor rendimiento, seguido por los porteros. Estas diferencias, pueden deberse al hecho que los jugadores adultos ya desarrollaron su potencial físico de manera más específica en función de las demandas del puesto durante el partido (Di Salvo et al., 2007), mientras que las jóvenes futbolistas de este estudio se encuentran en un proceso de formación.

Producto de la naturaleza de este primer estudio de tipo descriptivo, se sugiere que las próximas líneas de investigación puedan estar orientadas a; (1) estudiar el impacto del efecto de edad relativo sobre el rendimiento de las capacidades condicionantes dentro de una misma categoría; (2) profundizar sobre la relación que existe entre las variables antropométricas y el rendimiento deportivo en cada categoría; (3) relacionar el rendimiento de las diferentes pruebas, con el pico de velocidad de crecimiento (PVC).

Finalmente creemos que estas sugerencias podrán aportar un conocimiento de mayor relevancia a la disciplina y al contexto deportivo donde se origina este estudio.

\section{Conclusiones}

Los resultados obtenidos en la presente investigación sugieren que los atletas de las categorías más jóvenes Sub13, Sub-14 y Sub-15 son más altos que los registrados en la literatura. Las diferencias más significativas se presentaron entre las categorías Sub-13 y Sub-14, las cuales se encuentran mayormente afectadas por los estados de maduración de los jugadores en esa franja de edad (Malina et al., 2017).

Esto a su vez, se puede asociar al hecho que el $73,2 \%$ de los atletas de este estudio nacieron en el primer semestre del año de selección lo que deja de manifiesto la presencia del efecto de edad relativa.

Finalmente, la evidencia permite describir un perfil más completo de los jóvenes futbolistas chilenos en las diferentes categorías y posiciones, estos datos se deberían tener en cuenta en el proceso de selección, exclusión y permanencia de los atletas en las diferentes categorías en esta disciplina deportiva, como un indicador valido de referencia para los entrenadores.
De este modo, se creará un estándar de selección más objetivo y con un menor margen de error para los entrenadores. En este sentido es primordial seguir promoviendo la investigación en esta materia con la finalidad de fortalecer los hallazgos del presente estudio y promover las nuevas líneas de investigación.

\section{Limitaciones}

El presente estudio considero dos equipos de la primera división del fútbol chileno los cuales se ubican en Santiago, lo cual podría no ser representativo de los equipos del resto de la ciudad o mayor aún del resto de las regiones del país, considerando la diversidad geográfica, climatológica y cultural del país.

\section{Referencias}

Al Haddad. H., Simpson. B., Buchheit. M., Di Salvo. V. \& Mendez-Villanueva. A. (2015). Peak match speed and maximal sprinting speed in young soccer players: effect of age and playing position. Int J Sports Physiol Perform. 10(7). 888-896. doi:10.1123/ijspp.2014-0539

Andrade. V., Zagatto. A., Kalva. C., Mendes. O., Gobatto. C., Campos. E. \& Papoti. M. (2015). Running-based Anaerobic Sprint Test as a Procedure to Evaluate Anaerobic Power. International Journal of Sports Medicine. 36(14). 1156-1162. doi:10.1055/s-0035-1555935

Augste. C. \& Lames. M. (2011). The relative age effect and success in German elite U-17 soccer teams. Journal of Sports Sciences. 29(9). 983-987. doi:10.1080/ 02640414.2011.574719

Bangsbo. J., Iaia. F. \& Krustrup. P. (2008). The Yo-Yo intermittent recovery test - A useful tool for evaluation of physical performance in intermittent sports. Sports Medicine. 38(1). 37-51. doi:10.2165/00007256-20083801000004

Barnsley. H., Thompson. H. \& Barnsley. P. (1985). Hockey success and birthdate: The relative age effect. Canadian Association for Health. Physical Education. and Recreation. 51(1). 23-28.

Bergkamp. T., Niessen. S., den Hartigh. R., Frencken. W. \& Meijer. R. (2019). Methodological issues in soccer talent identification research. Sports Medicine. 49(9). 1317-1335. doi:10.1007/s40279-019-01113-w

Bernal-Orozco, M. F., Posada-Falomir, M., Quiñónez-Gastélum, C. M., Plascencia-Aguilera, L. P., Arana-Nuño, J. R., Badillo-Camacho, N., et al. (2020). Anthropometric and body composition profile of young professional soccer players. The Journal of Strength \& Conditioning Research, 34(7), 1911-1923.

Bolívara. J., Sandovalc. Ó., Osorioa. J., Dibd. G. \& Gallo. J. (2015). Relación entre la edad cronológica y la maduración sexual con la maduración ósea mediante resonancia magnética de la epífisis distal del radio en futbolistas adolescentes. Apunts Med Esport. 50(188). 129-137.

Burgess. K., Holt. T., Munro. S. \& Swinton. P. (2016). Reliability and validity of the running anaerobic sprint test (RAST) in soccer players. Journal of Trainology. 5(2). 24-29. 
Cipryan. L. \& Gajda. V.(2011). The influence of aerobic power on repeated anaerobic exercise in junior soccer players. Journal of Human Kinetics. 28. 63-71.

Cooke. C., Cobley. S., Till. K. \& Wattie. N. (2010). Searching for sporting excellence: talent identification and development. Journal of Sports Medicine. 44. doi:10.1136/ bjsm.2010.078725.220

Couto. L., Macedo. E., Melo. R., Teoldo. V.\& Fahel.A. (2009). Análise do Quartil de nascimiento de atletas porfessionais de fútbol. Pensar a Práctica. 12(3). 1-9.

Del Campo. D., Vicedo. J., Villora. S. \& Jordan. O. R. C. (2010). The relative age effect in youth soccer players from Spain. Journal of Sports Science \& Medicine. 9(2). 190.

Deprez. D., Coutts. A. J., Lenoir. M., Fransen. J., Pion. J., Philippaerts. R. \& Vaeyens. R. (2014). Reliability and validity of the Yo-Yo intermittent recovery test level 1 in young soccer players. Journal of Sports Sciences. 32(10). 903-910.

Deprez. D., Fransen. J., Lenoir. M., Philippaerts. R. \& Vaeyens. R. (2015). The Yo-Yo intermittent recovery test level 1 is reliable in young high-level soccer players. Biology of Sport. 32(1). 65.

Di Salvo, V., Baron, R., Tschan, H., Montero, F. C., Bachl, N., \& Pigozzi, F. (2007). Performance characteristics according to playing position in elite soccer. International Journal of Sports Medicine, 28(3), 222-227.

Dodd. K. \& Newans. T. (2018). Talent identification for soccer: Physiological aspects. Journal of Science and Medicine in Sport. 21(10). 1073-1078. doi:10.1016/ j.jsams.2018.01.009

Duarte. J. (2015). Perfil antropométrico del jugador de fútbol categoría Sub-16 de O'higgins de Rancagua. Revista Ciencias de la Actividad Física UCM. 16(2). 21-27.

Figueiredo. A., Silva. M., Cumming. S. \& Malina. R. (2010). Size and Maturity Mismatch in Youth Soccer Players 11to 14-Years-Old. Pediatric Exercise Science. 22(4). 596612. doi:10.1123/pes. 22.4 .596

González. J. (2007). El efecto relativo de la edad en el fútbol. Arch Med Deporte. 24(117). 5-13.

Grossmann. B. \& Lames. M. (2015). From Talent to Professional Football - Youthism in German Football. International Journal of Sports Science \& Coaching. 10(6). 1103-1113. doi:10.1260/1747-9541.10.6.1103

Gutierrez. D., Pastor. J., Gonzalez. S. \& Contreras. O. (2010). The relative age effect in youth soccer players from Spain. Journal of Sports Science and Medicine. 9. 190-198.

Hazir. T., Kose. M. \& Kin-Isler. A. (2018). The validity of running anaerobic sprint test to assess anaerobic power in young soccer players. Isokinetics and Exercise Science. 26(3). 201-209.

Hernández-Mosqueira. C., Fernandes. S., Fernandes. J., Retamales. F., Ibarra. J., Hernández-Vasquez. D. \& Valenzuela. R. (2013). Descripción de la composición corporal y somatotipo de futbolistas Sub-18. en función de la posición en el campo. Motricidad. European Journal of Human Movement. 31. 47-158.

Hernández. Y. \& García. J. (2014). Efectos de un entrenamiento de fuerza en futbolistas juveniles españoles. con la carga donde manifiestan el mejor valor de potencia en el ejercicio de salto cargado. Revista Iberoamericana de
Ciencias de la Actividad Física y el Deporte. 3(2). 9-17. Hill. B. \& Sotiriadou. P. (2016). Coach decision-making and the relative age effect on talent selection in football. European Sport Management Quarterly. 16(3). 292-315. doi:10.1080/16184742.2015.1131730

Jorquera. C., Rodríguez. F., Torrealba. M. \& Barraza. F. (2012). Composición Corporal y Somatotipo de Futbolistas Chilenos Juveniles Sub- 16 y Sub- 17. Int. J. Morphol. 30. 247-252.

Krustrup. P., Mohr. M., Amstrup. T., Rysgaard. T., Johansen. J., Steensberg. A., et al. (2003). The Yo-Yo intermittent recovery test: Physiological response. reliability. and validity. Medicine and Science in Sports and Exercise. 35(4). 697-705. doi:10.1249/01.Mss.0000058441.94520.32

Kunrath. C., Gonçalves. E., Teoldo. I. \& Barbosa. M. (2017). Maturação somática e aptidão física em jovens jogadores de futebol. Revista Andaluza de Medicina del Deporte. 10(4). 187-191. doi:10.1016/j.ramd.2016.01.002

Little. T. \& Williams. A. (2003). Specificity of acceleration. maximum speed and agility in professional soccer players. London: Routledge.

Little. T. \& Williams. A. (2005). Specificity of acceleration.maximum speed. and agility in professional soccer players. Journal of Strength and Conditioning Research. 19(1). 76-78.

López-Gómez, B., Pérez-Mendoza, D. A., Guzmán-Revelo, J. S., Rangel-Caballero, L. G., Corzo-Vargas, Y., de Paula Facioli, T., et al. (2020). Análisis del patrón de carrera sobre superficie artificial y natural en futbolistas adolescentes. Retos, 38, 109-113.

Los Arcos, A., \& Martins, J. (2018). Physical fitness performance of young professional soccer players does not change during several training seasons in a Spanish elite reserve team: Club Study, 1996-2013. The Journal of Strength \& Conditioning Research, 32(9), 2577-2583.

Malina. R., Figueiredo. A. \& Coelho-E-Silva. M. (2017). Body size of male youth soccer players: 1978-2015. Sports Medicine. 47(10). 1983-1992. doi:10.1007/s40279-0170743-x

Malý. T., Zahálka. F., Hráský. P., Mala. L., Izovská. J., Bujnovský. D., et al. (2015). Age-related differences in linear sprint and power characteristics in youth elite soccer players. Journal of Physical Education and Sport. 15(4). 857-863. doi:10.7752/jpes.2015.04132

Marqués, M. C., Izquierdo, M., Gabbett, T. J., Travassos, B., Branquinho, L. \& van den Tillaar, R. (2016). Physical fitness profile of competitive young soccer players: Determination of positional differences. International Journal of Sports Science \& Coaching, 11(5), 693-701.

Maureira, F. (2017). Estadística para educación física. Madrid: Bubok Publishing.

Maureira, F. \& Flores, E. (2018). Manual de investigación cuantitativa. Madrid: Bubok Publishing.

Nicholls. S. \& Worsfold. P. (2016). The observational analysis of elite coaches within youth soccer: The importance of performance analysis. International Journal of Sports Science \& Coaching. 11(6). 825-831. doi:10.1177/ 1747954116676109

Ostojic. S., Castagna. C., Calleja-González. J., Jukic. I., Idrizovic. K. \& Stojanovic. M. (2014). The biological age of 14- 
year-old boys and success in adult soccer: do early maturers predominate in the top-level game? Research in Sports Medicine. 22(4). 398-407.

Piqueras. P., Malavés. R. \& López. V. (2010). Seguimiento longitudinal de la evolución en la condición aeróbica en jóvenes futbolistas. Apunts. Medicina de l'Esport. 45(168). 227-234.

Rebelo, A., Brito, J., Maia, J., Coelho-e-Silva, M. J., Figueiredo, A. J., Bangsbo, J., et al. (2013). Anthropometric characteristics, physical fitness and technical performance of under-19 soccer players by competitive level and field position. Int J Sports Med, 34(4), 312-317.

Ribeiro. J., Teixeira. L., Lemos. R., Teixeira. A., Moreira. V., Silva. P. \& Nakamura. F. Y. (2019). Effects of Plyometric Versus Optimum Power Load Training on Components of Physical Fitness in Young Male Soccer Players. International Journal of Sports Physiology and Performance. 1(aop). 1-9.

Roca. L., Vásquez. P. \& Valderas. L. (2017). Valoración del pico de velocidad de crecimiento y estatura prevista definitiva de acuerdo a las posiciones de juego de los futbolistas Sub - 15 y 16 de Rangers de Talca. Revista Digital de Educación Física. 46. 107-115.

Roca. L., Vásquez. P., Valderas. L., Sepúlveda. V. \& González. M. (2018). Valoración de la potencia y el índice de fatiga de los futbolistas Sub-15 y 16 de Rangers de acuerdo a las posiciones de juego. Revista de Entrenamiento Deportivo. 31(4).

Sarmento. H., Anguera. M. T., Pereira. A. \& Araujo. D. (2018). Talent identification and development in male football: a systematic review. Sports Med. 48(4). 907-931. doi:10.1007/ s40279-017-0851-7

Sayers. S., Harackiewicz. D., Harman. E., Frykman. P. \& Rosenstein. M. (1999). Cross-validation of three jump power equations. Medicine and Science in Sports and Exercise.31(4). 572-577. doi:10.1097/00005768-19990400000013
Schmitz. B., Pfeifer. C., Kreitz. K., Borowski. M., Faldum.A. \& Brand. S. (2019). Normative Yo-Yo Intermittent Recovery Level 1 and Yo-Yo Intermittent Endurance Level 1 test values of boys aged 9-16 years. Journal of Science and Medicine in Sport. 22(9). 1030-1037. doi:10.1016/ j.jsams.2019.05.016

Silva-Junior. C., Palma. A., Costa. P., Pereira-Junior. P., Barroso. R., Abrantes-Junior, R. \& Barbosa, M. (2011). Relação entre as potências de sprint e salto vertical em jovens atletas de futebol. Motricidade. 7(4). 5-13.

Silva. M., Figueiredo. A., Moreira. H. \& Malina. R. (2008). Functional capacities and sport-specific skills of 14- to 15-year-old male basketball players: Size and maturity effects. European Journal of Sport Science. 8(5). 277285. doi:10.1080/17461390802117177

Skorski. S., Skorski. S., Faude. O., Hammes. D. \& Meyer. T. (2016). The relative age effect in elite German youth soccer: Implications for a successful career. International Journal of Sports Physiology and Performance. 11(3). 370-376.

Toro. A. (2001). Análisis fisiológico del esfuerzo físico según el puesto del jugador de fútbol. Disponible en: https:/ /g-se.com/analisis-fisiologico-del-esfuerzo-fisico-segunel-puesto-del-jugador-de-futbol-78-sa-657cfb270ef7ad

Unnithan. V., White. J., Georgiou. A., Iga. J. \& Drust. B. (2012). Talent identification in youth soccer. J Sports Sci. 30(15). 37-41. doi:10.1080/02640414.2012.731515

Vaeyens. R., Lenoir. M., Williams. A. \& Philippaerts. R. (2008). talent identification and development programmes in sport. Sports Medicine. 38(9). 703-714.

Van den Honert. R. (2012). Evidence of the relative age effect in football in Australia. Journal of Sports Sciences. 30(13). 1365-1374. doi:10.1080/02640414.2012.707329

Vincent. J. \& Glamser. F. D. (2006). Gender differences in the relative age effect among US Olympic Development Program youth soccer players. Journal of Sports Sciences. 24(4). 405-413. doi:10.1080/02640410500244655

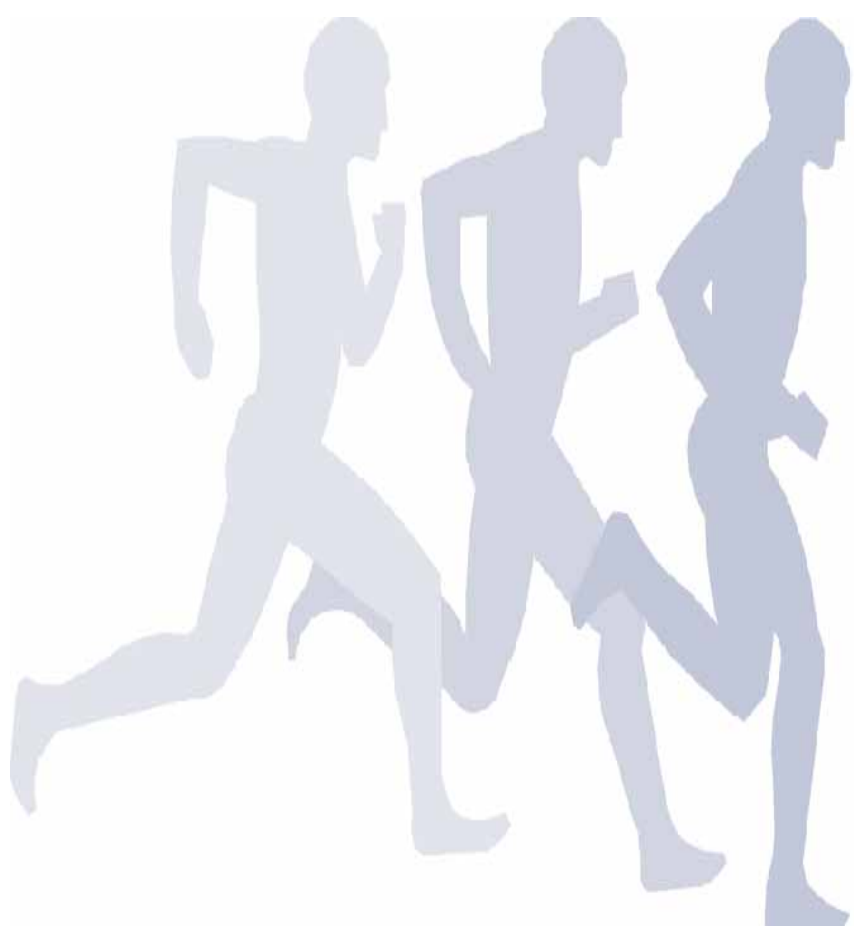

